\title{
Clinicopathological Characteristics of Gastric-type Endocervical Adenocarcinoma Misdiagnosed as an Endometrial, Ovarian or Extragenital Malignancy, or Mistyped as Usual-type Endocervical Adenocarcinoma
}

\author{
HYUN HEE KOH ${ }^{1}$, YOON YANG JUNG ${ }^{2}$ and HYUN-SOO KIM ${ }^{1}$ \\ ${ }^{1}$ Department of Pathology and Translational Genomics, Samsung Medical Center, \\ Sungkyunkwan University School of Medicine, Seoul, Republic of Korea; \\ ${ }^{2}$ Department of Pathology, Myongji Hospital, \\ Hanyang University College of Medicine, Goyang-si, Republic of Korea
}

\begin{abstract}
Background/Aim: The diagnosis of gastric-type endocervical adenocarcinoma (GEA) is challenging because its differential diagnosis includes not only gynecological tumors, but also extragenital tumors. Patients and Methods: We reviewed the electronic medical records and all available slides to investigate the clinicopathological characteristics of eight misdiagnosed GEA cases. Results: Three tumors were initially misdiagnosed as endometrial carcinoma. They displayed extensive endomyometrial involvement and complex glandular architecture, but no severe nuclear pleomorphism. Another three tumors were misclassified as usual-type endocervical adenocarcinoma because of mucin-poor, pseudoendometrioid glands, apical mitotic figures, and karyorrhectic debris. The two remaining tumors presenting as adnexal masses mimicked primary ovarian mucinous tumor and metastatic cholangiocarcinoma. Conclusion: The varying pathological characteristics of GEA reflect the variability in clinical manifestations and its diagnostic difficulties. It is challenging to make an accurate diagnosis based solely on
\end{abstract}

This article is freely accessible online.

Correspondence to: Hyun-Soo Kim, Department of Pathology and Translational Genomics, Samsung Medical Center, Sungkyunkwan University School of Medicine, 81, Irwon-ro, Gangnam-gu, Seoul 06351, Republic of Korea. Tel: +82 234101243, Fax: +82 234142831, e-mail: hyun-soo.kim@samsung.com; Yoon Yang Jung, Department of Pathology, Myongji Hospital, Hanyang University College of Medicine, 14beon-gil, Hwasu-ro, Goyang-si, Gyeonggido 10475, Republic of Korea. Tel: +82 318107101, Fax: +82 318107109, e-mail: jy@mjh.or.kr

Key Words: Uterus, cervix, gastric-type endocervical adenocarcinoma, misdiagnosis, mistyping. histological features. When suspecting GEA, clinicians should consider more comprehensively the clinicopathological context, along with immunostaining results.

Although cervicovaginal cytology screening has decreased the incidence of cervical carcinoma and its associated mortality, cervical carcinoma remains the fourth most common gynecological malignancy worldwide. Adenocarcinoma of the uterine cervix is relatively less common than squamous cell carcinoma. Despite the decreased incidence of cervical carcinoma, the proportion of endocervical adenocarcinoma is steadily increasing (1). The recently proposed International Endocervical Adenocarcinoma Criteria and Classification (IECC) divides endocervical adenocarcinomas into human papillomavirus (HPV)-associated adenocarcinomas (HPVA) and HPV-independent adenocarcinomas (2). Most endocervical adenocarcinomas [i.e., usual-type endocervical adenocarcinomas (UEAs) and mucinous-type adenocarcinomas] are causally related to oncogenic infection with high-risk HPV (3). Meanwhile, HPV-independent adenocarcinomas include gastrictype, mesonephric-type, clear cell-type adenocarcinomas. They are relatively rare and often represent diagnostic or management challenges with their distinct morphology and molecular genomics and different responses to standard therapies.

Gastric-type endocervical adenocarcinoma (GEA) is the most common HPV-independent adenocarcinoma of the cervix. Initially, it was included as a distinctive variant of mucinous carcinoma in the 2014 World Health Organization (WHO) Classification of Tumours of Female Reproductive Organs (4). However, it has been re-classified as one of the HPV-independent subtypes in the latest 2020 WHO Classification (5). Its recognition has provoked the abovementioned discussion on dividing endocervical adenocarcinomas into two groups according to the association 
with HPV (6-8), because GEA presents specific histological features and immunophenotype, as well as aggressive clinical behavior. This tumor is morphologically characterized by irregular and dilated glands; abundant, clear, or pale eosinophilic cytoplasm; distinct cell borders; varying degrees of nuclear pleomorphism; and prominent stromal desmoplasia. Recent studies have consistently shown that GEA is diagnosed at a clinically advanced stage and is usually more advanced than HPVA. Further, GEA displays significantly worse prognosis than HPVA (8-11).

Endocervical adenocarcinoma can be easily diagnosed to be of cervical origin when it is confined to the cervix. However, distinguishing it from endometrial or ovarian carcinoma is challenging due to their overlapping morphologies and immunoprofiles, particularly when the tumor extends to the uterine corpus or adnexa or when a precursor lesion is not distinct (12). Given that most uterine adenocarcinomas involving both the cervix and corpus are of endometrial origin, endocervical adenocarcinomas that extensively involve the uterine corpus can be misdiagnosed as endometrial carcinoma (13).

We recently encountered several cases of GEA that were either initially misdiagnosed as endometrial carcinoma, ovarian carcinoma, or metastatic carcinoma of extragenital origin, or mistyped as UEA. Awareness of these misdiagnoses prompted us to initiate a comprehensive clinicopathological review. We investigated the clinicopathological characteristics and immunostaining results of eight misdiagnosed cases of GEA.

\section{Patients and Methods}

Patient selection. This study (2020-11-043) was approved by the Institutional Review Board of Samsung Medical Center (Seoul, Republic of Korea) and was conducted according to the tenets of the 1964 Declaration of Helsinki and its later amendments. Eight patients with GEA were identified from the surgical pathology archives. The following clinical information was collected from the electronic medical records: reason for the study inclusion; age; past history of gynecological disease; presenting symptom or sign; preoperative diagnosis of endometrial curettage or cervical punch biopsy; preoperative clinical impression; surgical procedure; intraoperative frozen section diagnosis of salpingooophorectomy specimen; final pathological diagnosis of hysterectomy specimen; International Federation of Gynecology and Obstetrics (FIGO) stage; postoperative treatment; and current status.

Slide review. Two board-certified pathologists specialized in gynecological oncology examined all available hematoxylin and eosin-stained slides via light microscopy. The following pathological information was collected: greatest dimension and invasion depth of tumor; extension to the endomyometrium, parametrium, uterine serosa, vagina, and adnexa; lymphovascular space invasion (LVSI); and metastasis to the lymph node, peritoneum, and distant organ. We chose the most representative slide for each case to perform immunostaining.
Immunohistochemical staining. Immunostaining was performed using an automatic instrument (Ventana Benchmark XT, Ventana Medical Systems, Oro Valley, AZ, USA) (14-24). Antigen retrieval was performed using Cell Conditioning Solution (CC1, Ventana Medical Systems). The 4- $\mu$ m-thick formalin-fixed, paraffin embedded sections were incubated with primary antibodies against p16 (prediluted, clone E6H4, Ventana Medical Systems), p53 (1:300, clone DO-7, Novocastra, Leica Biosystems, Newcastle Upon Tyne, UK), and Wilms tumor 1 (WT1, 1:200, clone 6F-H2, Cell Marque, Rocklin, CA, USA). After chromogenic visualization using an ultraView Universal DAB Detection Kit (Ventana Medical Systems), sections were counterstained with hematoxylin. Appropriate positive and negative controls were concurrently stained to validate the staining method. Endometrial serous carcinoma showing diffuse and strong immunoreactivities for p53 and ovarian high-grade serous carcinoma showing diffuse and strong immunoreactivities for WT1 and p16 were used as positive controls. The negative control was prepared by substituting nonimmune serum for primary antibody, resulting in no detectable staining.

The p53 immunostaining pattern was interpreted as a mutant pattern when one of the following staining patterns was observed: diffuse and strong nuclear immunoreactivity in $75 \%$ or more of the tumor cells (over-expression pattern); no nuclear immunoreactivity in any of the tumor cells (complete absence pattern); and an unequivocal cytoplasmic staining (cytoplasmic pattern) (25). In contrast, p53 expression was interpreted as a wild type pattern if a variable proportion of tumor cell nuclei expressed p53 protein with mild-tomoderate staining intensity $(15,26-28)$. The p16 immunostaining pattern was interpreted as block positive when p16 expression was continuous and strong, nuclear, or nuclear plus cytoplasmic staining. All other p16 immunostaining patterns, described as focal nuclear staining or wispy, blob-like, puddled, or scattered cytoplasmic staining, were interpreted as patchy positive (27-30).

\section{Results}

Clinical characteristics. The median patient age was 54 years (range $=41-72$ years). Table I summarizes the clinical characteristics. Overall, 7/8 (87.5\%) patients had no previous gynecological history, and 1 patient had previous history of uterine leiomyoma. There were 1 (12.5\%), 2 (25.0\%), and 5 $(62.5 \%)$ patients who underwent subtotal, total, and radical hysterectomy, respectively. Meanwhile, 1 (12.5\%) and 6 (75.0\%) patients underwent left and bilateral salpingooophorectomies, respectively. There were 4 (50.0\%) patients who underwent pelvic lymph node dissection and 1 (12.5\%) patient who underwent pelvic sentinel lymph node biopsy.

Three of the eight patients (patients 1-3) were initially misdiagnosed as having endometrial carcinoma. In these patients, the preoperative clinical impression was endometrial carcinoma. The initial diagnoses of endometrial curettage specimens were endometrioid (2/3; patients 1 and $2)$ and mucinous (1/3; patient 3$)$ carcinomas of the endometrium. In the latter case, the tumor involving both the endocervix and endometrium in the hysterectomy specimen was also misinterpreted as endometrial mucinous carcinoma.

Meanwhile, the histological type of the other three patients (patients 4-6) was mistakenly determined as UEA. 


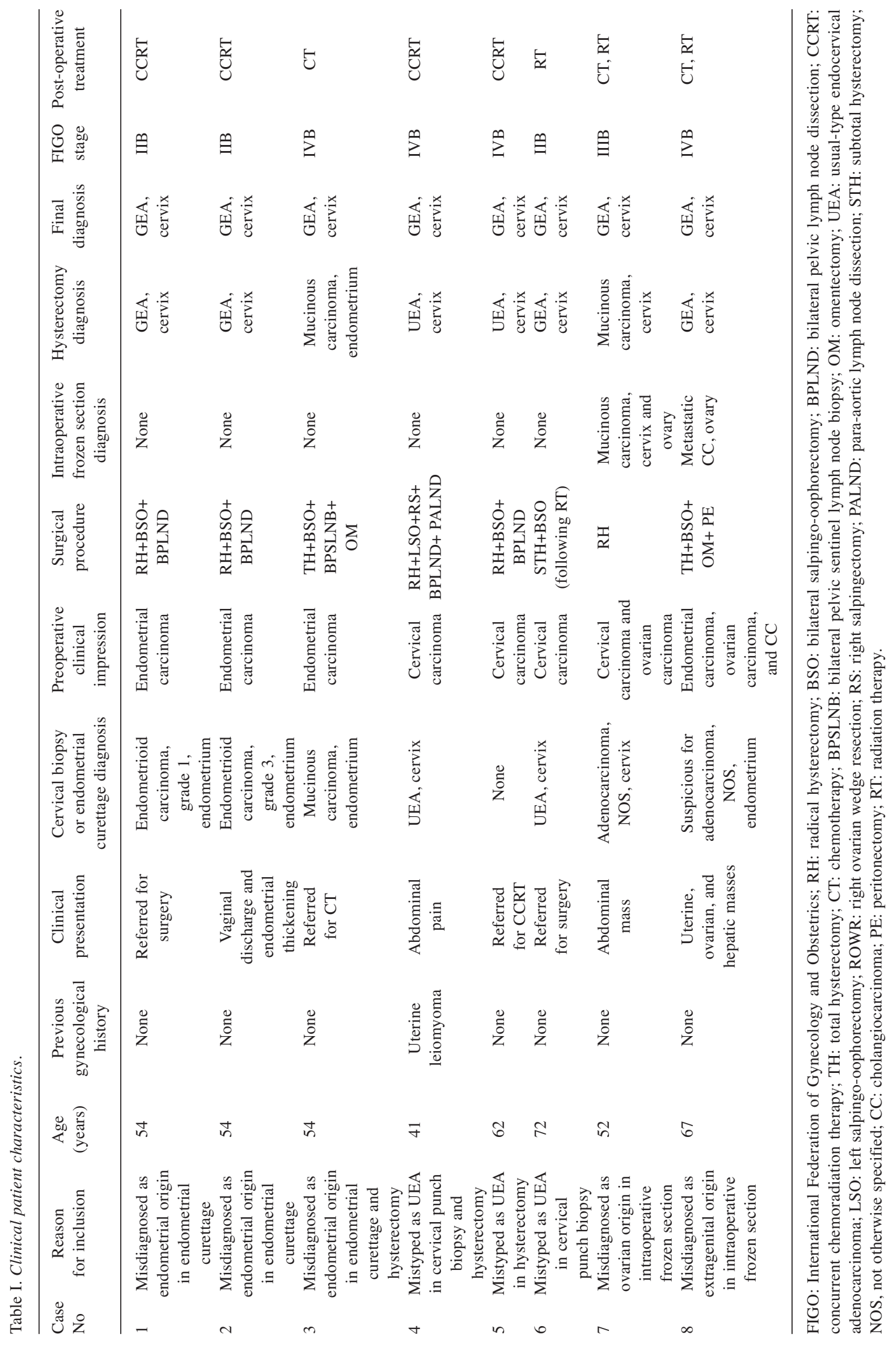


In two patients (patients 4 and 5), radical hysterectomy specimens were misinterpreted as UEA. One of these two patients developed distant metastases in the supraclavicular lymph nodes, while the other developed metastases in the colon. The biopsy specimens obtained from the metastatic lesions were correctly diagnosed as GEA. One patient (patient 6) who was initially diagnosed with UEA in her cervical punch biopsy underwent radiation therapy before surgery. The surgeons failed to perform radical hysterectomy because of severe fibrosis and adhesion due to the preoperative radiation therapy. She underwent a subtotal hysterectomy with bilateral salpingo-oophorectomy.

The remaining two patients (patients 7 and 8) had adnexal tumors in addition to the uterine tumors. One patient (patient 7) was preoperatively suspected to have concurrent ovarian and uterine carcinomas. She was initially diagnosed with adenocarcinoma, not otherwise specified (NOS), on both the cervical punch biopsy and endometrial curettage. Given that she had a huge cystic ovarian mass that was radiologically compatible with primary ovarian carcinoma, the intraoperative frozen section diagnosis of ovarian-origin mucinous carcinoma involving the ovary and cervix was made. Similar to the former patient, cervical punch biopsy and endometrial curettage diagnoses of the latter patient (patient 8) suggested adenocarcinoma, NOS. Considering that she had multiple masses in the liver, uterus, and bilateral ovaries, the intraoperative frozen section diagnosis of ovarian tumor was metastatic cholangiocarcinoma.

All patients had advanced-stage cervical carcinoma. The FIGO stage was IIB in $37.5 \%$ (3/8; patients 1,2 , and 6$)$ of the patients; IIIB, $12.5 \%$ (1/8; patient 7$)$; and IVB, $50.0 \%$ (4/8; patients $3,4,5$, and 8$)$. There were 4 (50.0\%; patients $1,2,4$, and 5) and 2 (25.0\%; patients 7 and 8$)$ patients who underwent postoperative concurrent chemoradiation therapy and radiation therapy after chemotherapy, respectively. Each of the two remaining patients underwent radiation therapy (patient 6) and chemotherapy (patient 3) after surgery.

Pathological characteristics. Table II summarizes the pathological characteristics. The greatest dimension ranged from $23 \mathrm{~mm}$ to $60 \mathrm{~mm}$ (median, $35 \mathrm{~mm}$ ). The invasion depth ranged from $3 \mathrm{~mm}$ to $16 \mathrm{~mm}$ (mean, $11.4 \mathrm{~mm}$ ). Extensive endomyometrial involvement was observed in all patients. All except $1(7 / 8,87.5 \%)$ tumor involved the entire thickness of the cervical stroma and extended into the parametrium and uterine serosa. Vaginal and adnexal involvement was identified in 4 $(50.0 \%)$ and $5(62.5 \%)$ patients, respectively. There were 5 (62.5\%) patients who showed LVSI, and pelvic lymph node metastases were detected in 3 of these 5 patients. Meanwhile, 2 patients $(25.0 \%)$ had pelvic peritoneal metastases at the time of surgery. Moreover, 4 patients $(50.0 \%)$ had distant metastases to the liver (2/8; patients 3 and 8$)$, bone (1/8; patient 4$)$, colon ( $1 / 8$; patient 4$)$, and supraclavicular lymph node (1/8; patient 5$)$.

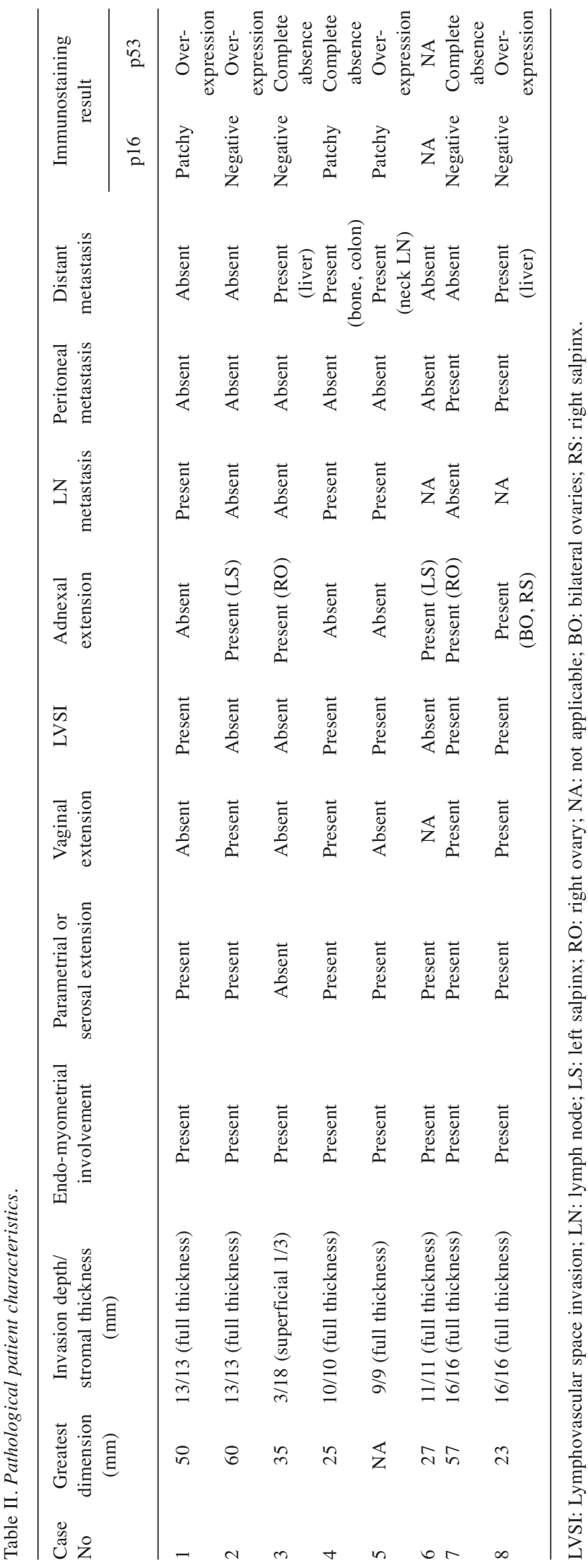




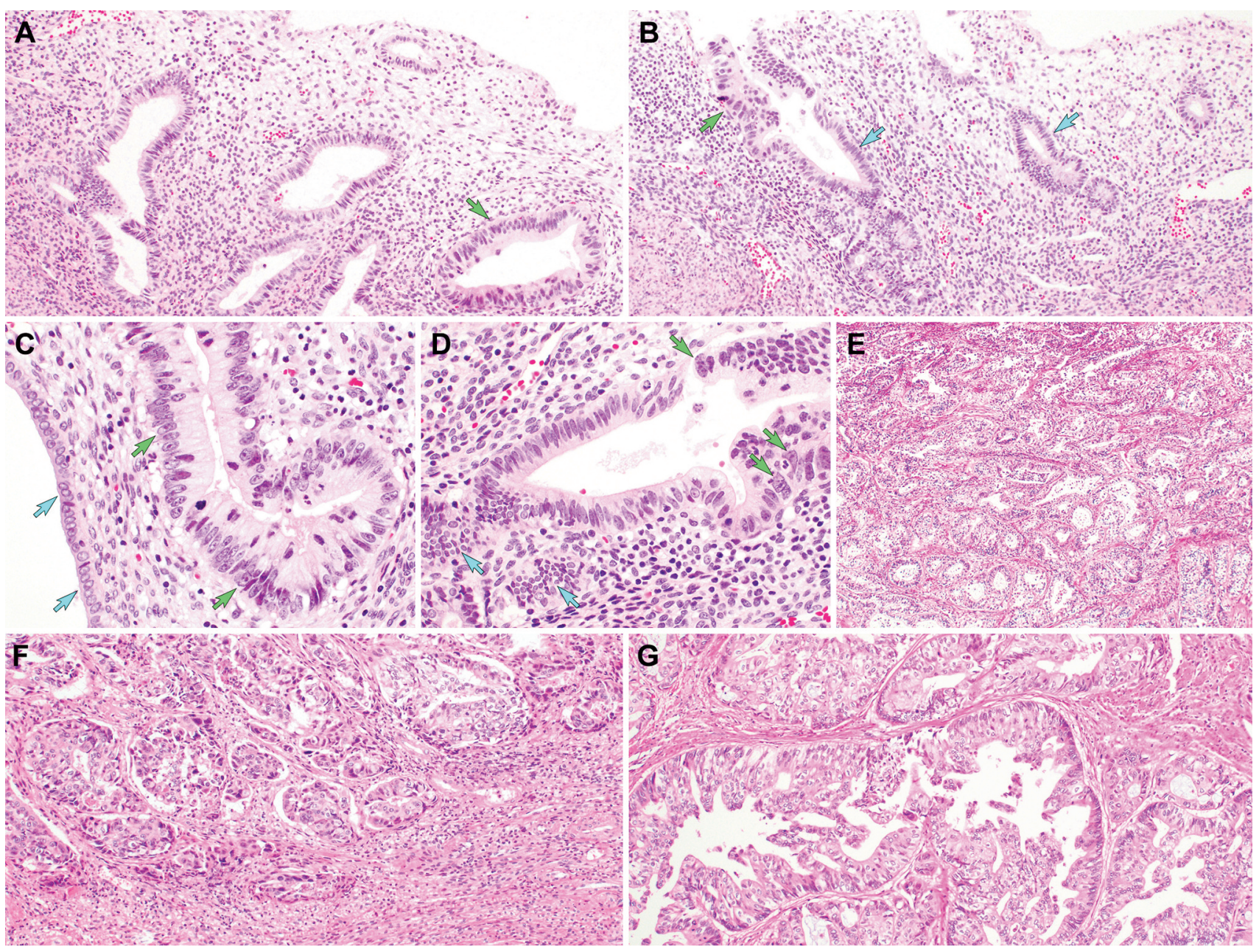

Figure 1. Gastric-type endocervical adenocarcinoma mimicking endometrial atypical hyperplasia/endometrioid intraepithelial neoplasia (AH/EIN) and endometrioid carcinoma. (A) At low-power magnification, a gland in the right lower corner (green arrow) shows nuclear enlargement and hyperchromasia. (B) A slightly enlarged gland (green arrow) shows loss of nuclear polarity as well as significant nuclear enlargement and pleomorphism than does the adjacent non-atypical glandular epithelium (blue arrows). Obvious cytological demarcation but without stromal invasion raises the suspicion of endometrial AH/EIN. (C) High-power view reveals atypical glandular epithelium showing enlarged nuclei with coarse chromatin and conspicuous nucleoli (green arrows). The adjacent non-atypical gland shows a single layer of inactive cuboidal epithelium with small, bland-appearing nuclei (blue arrows). (D) The pleomorphic nuclei (green arrows) partially occupies the endometrial gland. Note the cytological demarcation between atypical (upper right corner) and non-atypical (lower left corner) cells (blue arrows). (E) The tumor tissue consists predominantly of crowded glands and thin intervening stroma, resembling low-grade endometrioid carcinoma of the endometrium. $(F)$ The invasive tumor front shows minimal inflammatory infiltrates without stromal desmoplasia. $(G)$ There are several foci of glandular dilatation, intraluminal papillary projections, and cribriform architecture, closely similar to the features of endometrioid carcinoma. Staining method: A-G, hematoxylin and eosin staining. Original magnification: A, 100x; B, 100x; C, 400x; D, 400x; E, 40x; F, 100x; G, 100x.

We noted that the tumors misinterpreted as endometrial endometrioid carcinoma (patients 1 and 2; Figure 1) and UEA (patients 4-6; Figure 2) consisted mainly of mucin-depleted glands and showed minimal desmoplastic stromal response. Particularly, in patients 1 and 2, normal endometrial glandular epithelium was partially replaced by tumor cells, resembling endometrial atypical hyperplasia/endometrioid intraepithelial neoplasia (AH/EIN). Areas showing nuclear pseudostratification, hyperchromasia, and mild-to-moderate pleomorphism resembled those observed in endometrial $\mathrm{AH} / \mathrm{EIN}$ or low-grade endometrioid carcinoma. In both patients, the neoplastic glands exhibited no or little intracytoplasmic mucin. Patients 4-6 showed complex and cribriform glands deeply infiltrating the outer half of cervical stroma, deep myometrium, and parametrium. Even though most of them were associated with fibromyxoid stroma and prominent desmoplasia, the tumor cells possessed minimal intracytoplasmic mucin and did not exhibit gastric differentiation. Instead, several microscopic foci showed 


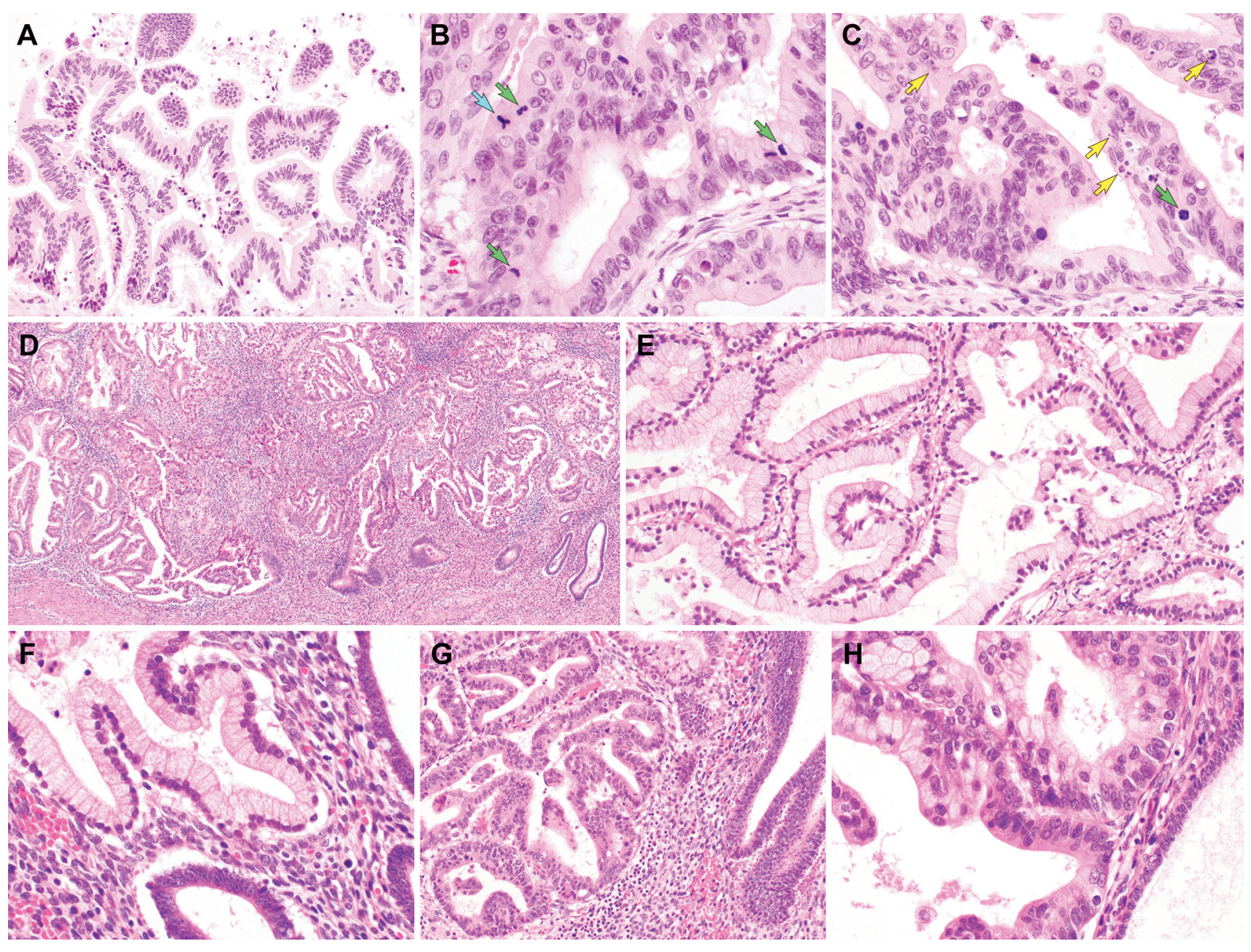

Figure 2. Gastric-type endocervical adenocarcinoma mimicking usual-type endocervical adenocarcinoma and endometrial mucinous adenocarcinoma. (A) The neoplastic glands exhibit architectural complexity with papillary fronds. The papillary structures fuse to form a network of anastomosing strands of tall columnar epithelial cells. The tumor cells have hyperchromatic, elongated nuclei, most of which are basally located. The intracytoplasmic mucin is not readily identified. (B) The tumor cells have variable amounts of eosinophilic cytoplasm. Enlarged, fusiform, hyperchromatic, pseudostratified nuclei show irregular, coarse chromatin with occasional prominent nucleoli. Apical or "floating" mitoses (green arrows) as well as an atypical mitotic figure (blue arrow) are present. (C) In addition to the mitotic figures (green arrow), frequent apoptotic bodies (yellow arrows) are also noted within the epithelium. The atypical mitotic figures and apoptotic bodies are typical histological features of usualtype endocervical adenocarcinoma. $(D)$ Low-power view reveals complex glandular proliferations involving the endometrial stroma and superficial myometrium. Crowded, branching glands resemble those of endometrial endometrioid carcinoma. A few normal endometrial glands are noted in the right lower corner. (E) The tumor cells have small, basally located nuclei with minimal-to-mild pleomorphism. Dilated glands are lined with columnar epithelial cells with abundant apical mucin. $(F-H)$ The tumor shows varying degrees of cytological atypia: $(F)$ a single layer of mucincontaining epithelium showing low-grade nuclear atypia, $(G)$ significant nuclear stratification and intermediate-grade nuclear atypia in complex glandular architecture, and $(H)$ mucin-containing epithelium high-grade nuclear atypia including loss of polarity, moderate-to-severe pleomorphism, and occasional prominent nucleoli. Staining method: A-H, hematoxylin and eosin staining. Original magnification: A, 100x; B, 400x; C, 400x; $D, 40 x ; E, 100 x ; F, 400 x ; G, 100 x ; H, 400 x$.

increased mitotic activity, karyorrhectic debris, and intraluminal necrotic debris, supportive of HPVA (i.e., UEA).

In patient 3 (Figure 2), the tumor consisted predominantly of confluent and dilated glands lined by mucin-containing cells with mild-to-moderate nuclear atypia. Further, it involved the superficial cervical stroma and inner half of the myometrium. The crowded glands with a complex branching architecture resembled those of low-grade endometrioid carcinoma. Interestingly, the degree of nuclear pleomorphism ranged from absent or slight to moderate. The location of epicenter in the endometrial cavity, lack of severe or marked nuclear pleomorphism, minimal desmoplastic stromal reaction, and superficial myometrial invasion were considered to support the diagnosis of low-grade mucinous carcinoma of the endometrium. 


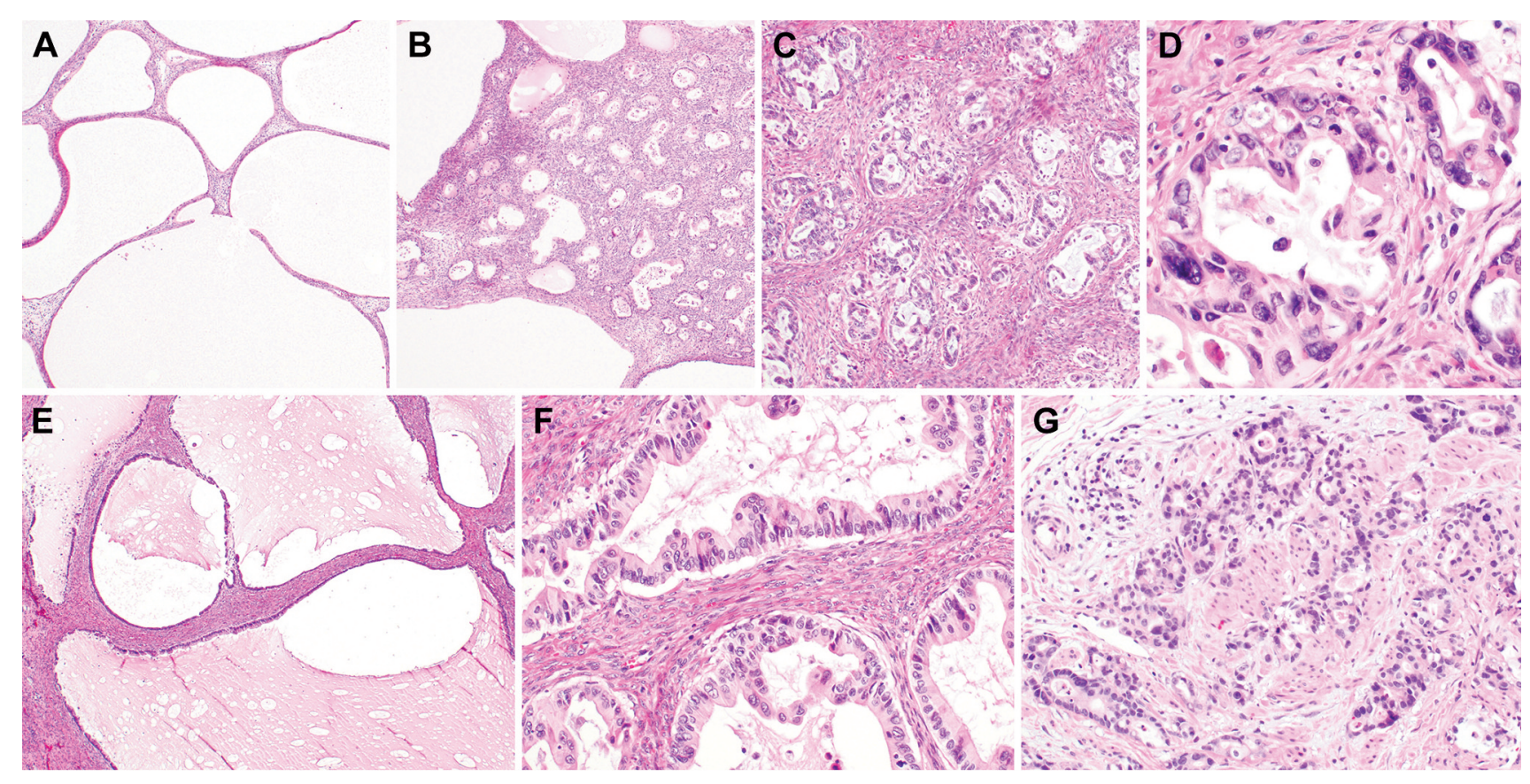

Figure 3. Ovarian metastasis of gastric-type endocervical adenocarcinoma mimicking primary ovarian mucinous carcinoma and metastatic cholangiocarcinoma. (A) Low-power view of patient 7 sample shows cystically dilated glands with thin intervening stroma. (B) There are some foci showing proliferation of small well-formed glands of various shapes. (C) They are relatively evenly distributed and separated by distinct fibrous stroma. Stromal inflammatory infiltrates and desmoplastic reaction are minimal. (D) The tumor cells often show marked nuclear pleomorphism. (E) Similar to patient 7, low-power view of patient 8 sample reveals that the tumor contains cystic spaces filled with eosinophilic mucinous material and lined by mucinous epithelium. $(F)$ Most of the lining epithelium exhibits high-grade nuclear atypia, and the intervening stroma appears similar to that of normal ovarian stroma. $(G)$ Back-to-back arrangements of small tubular glands as well as fused and interconnected poorly formed glands and tumor cell nests in an abundant fibrous stroma raises the suspicion of metastatic cholangiocarcinoma. Staining method: A-G, hematoxylin and eosin staining. Original magnification: A, 40x; B, 40x; C, 100x; D, 400x; E, 40x; F, 200x; G, 200x.

In two patients (patients 7 and 8; Figure 3) presenting with adnexal tumors, imaging findings of huge ovarian masses confused the pathologists. There were several scattered foci showing fused and interconnected mucinous glands in a confluent glandular pattern. Both tumors consisted dominantly of large, cystically dilated glands with nuclear stratification, intraluminal papillary growth, and mild-to-moderate nuclear pleomorphism. This raised the suspicion of ovarian mucinous borderline tumor or carcinoma. In addition to the expansile invasive pattern, the tumor in patient 7 also presented with multiple areas of destructive stromal invasive pattern showing relatively well-formed glands of various shapes and sizes, lined by atypical mucinous epithelium. The tumor cells often showed marked nuclear pleomorphism. Some goblet cells were randomly scattered between the mucin-containing epithelial cells. Similarly, in patient 8 , the intraoperative frozen section of the right ovarian mass also represented well-differentiated, small angulated, or cribriform glands with varying degrees of cytological atypia and nuclear pleomorphism in an abundant fibrous stroma, characteristic of cholangiocarcinoma.

In addition, multifocal microscopic metastases confined within the tubal surface epithelium were observed in patient
2 (Figure 4). The tumor cells proliferated along the fimbrial surface epithelium. Abrupt transitions between normal tubal epithelium and neoplastic glandular epithelium were noted in the fimbria, infundibulum, and ampulla. A few tiny tumor cell clusters (i.e., microscopic tumor emboli) were detected within the tubal lumina. The tumor cells possessed eccentrically located nuclei showing stratification, enlargement, and moderate-to-severe pleomorphism, with conspicuous nucleoli and occasional mitoses. A few intracytoplasmic vacuoles were observed on the apical surface. Microscopic segmental replacement of the fimbrial serous-type epithelium with atypical cells showing nuclear hyperchromasia and pleomorphism as well as mitotic figures closely resembled serous tubal intraepithelial carcinoma (STIC).

Despite the aforementioned histological features that could lead to misdiagnoses, all tumors showed variable amounts of typical histological features of GEA. This was characterized by crowded and irregular-shaped glands with stratification of cells that often protruded into the glandular lumina. The amount of intracytoplasmic mucin varied between different tumor areas. The tumor cells showed distinct cell borders and clear or pale eosinophilic and voluminous cytoplasm 


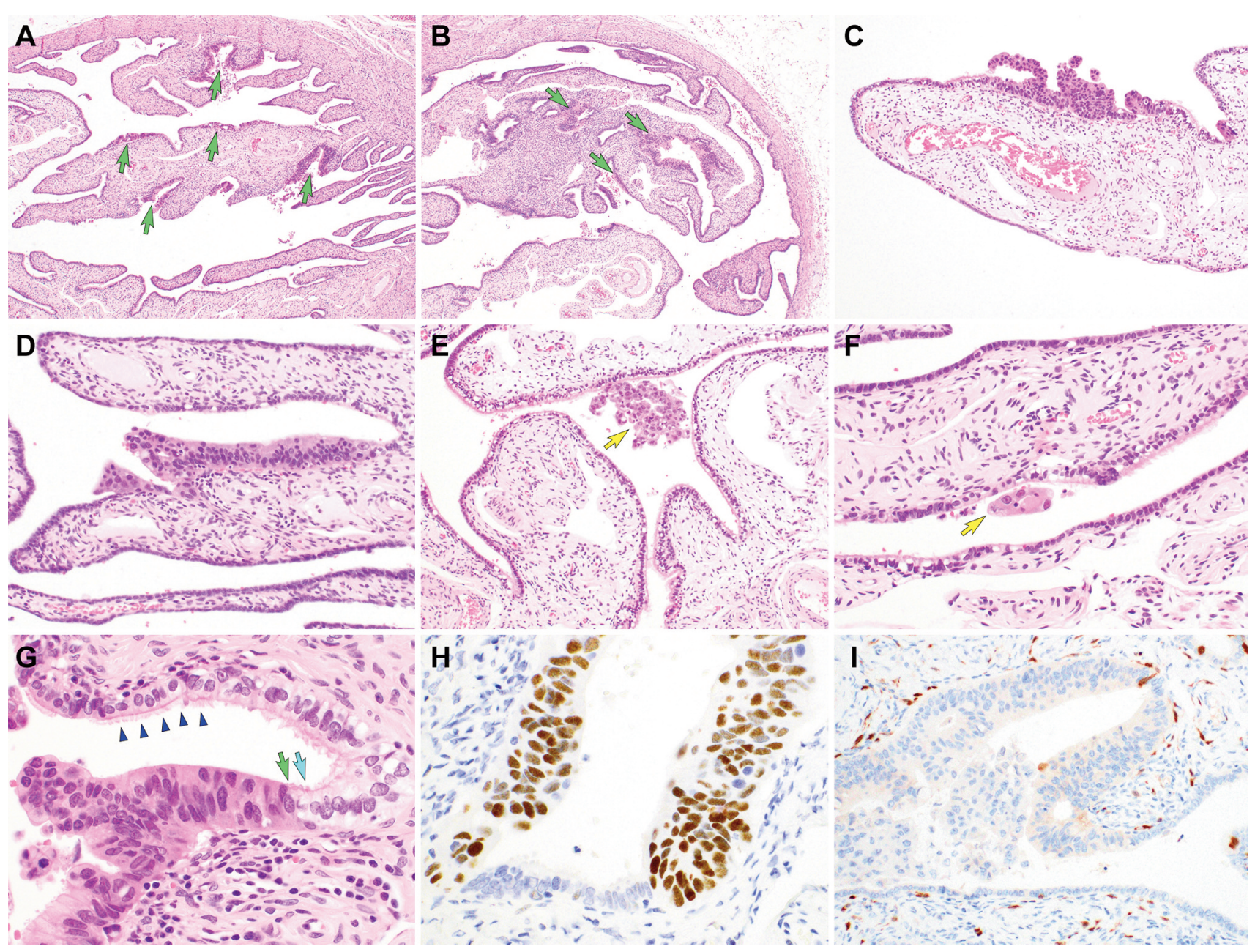

Figure 4. Tubal intraepithelial metastasis of gastric-type endocervical adenocarcinoma (GEA). (A-B) The microscopic metastatic tumors (green arrows) involve multifocally non-fimbriated portions of the fallopian tubes. (C-D) Fimbrial involvement is also noted among the tumor cells that spread horizontally along the surface epithelium. Nuclear pleomorphism and stratification, micropapillary structure, and abrupt transition between normal tubal epithelium and tumor cells are noted. (E-F) A few tiny intraluminal tumor emboli (yellow arrows) are present. (G) There are abrupt transitions between normal tubal epithelium (blue arrow) and tumor cells (green arrow). Navy arrowheads indicate ciliated normal tubal epithelium. (H-I) Metastatic GEA exhibits (H) p53 over-expression or (I) non-block p16 immunoreactivity. The tumor cells show faint cytoplasmic p16 expression only. Staining method: A-G, hematoxylin and eosin staining; H-I, polymer method. Original magnification: A, 40x; B, 40x; C, 100x; D, 150x; E, 150x; F, 200x; G, 400x; H, 400x; I, 200x.

with differing amounts of intracytoplasmic mucins. The tumor cell nuclei were basally located and ranged from small, round, or ovoid to markedly enlarged and irregular with vesicular chromatin and prominent nucleoli. Although some areas exhibited well-formed glands with minimal desmoplastic stromal reaction, most of the tumor tissues were moderately-to-poorly differentiated, consisting of infiltrating poorly formed glands, tumor nests, or single cells. Prominent stromal desmoplasia and inflammatory infiltrates were associated with the tumor.

Immunostaining results. Seven patients were available for immunostaining (Figure 5); of them, four patients (patients
1, 2, 5, and 8) exhibited p53 over-expression pattern, indicating missense tumor protein 53 (TP53) mutation. More than $90 \%$ of the tumor cell nuclei showed uniform nuclear p53 immunoreactivity with strong staining intensity. In patient 2, microscopic foci of tubal intraepithelial metastasis were diffusely and strongly positive for p53, whereas they were negative for WT1, highlighting the adjacent normal tubal epithelium. These immunophenotypes confirmed the diagnosis of metastatic GEA and excluded the possibility of STIC. The remaining three patients displayed complete absence of p53 immunoreactivity, indicating nonsense or frameshift TP53 mutation. None of the tumor cells showed p53 expression, whereas the nuclei of some normal 


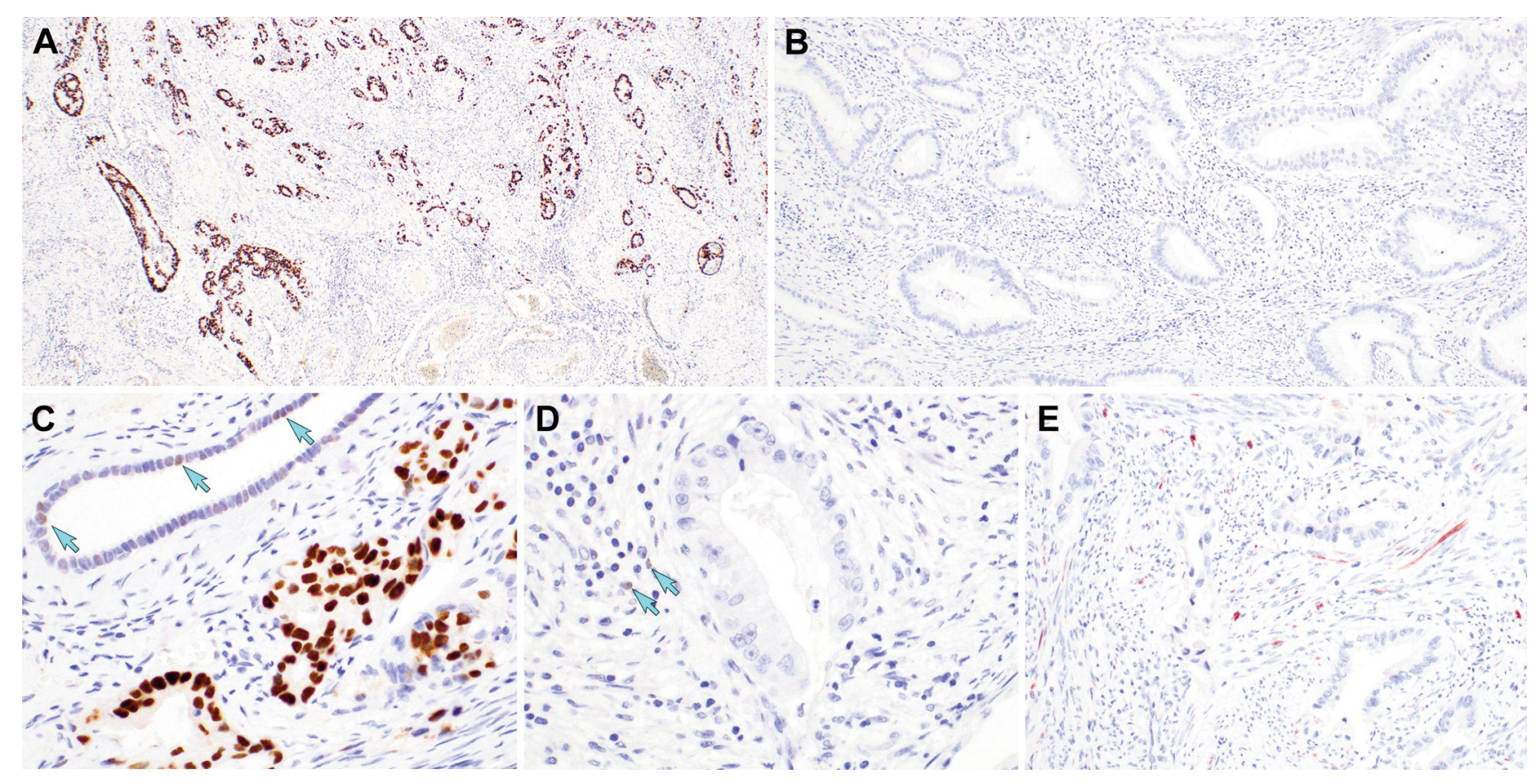

Figure 5. Immunophenotype of gastric-type endocervical adenocarcinoma. (A-B) Mutant p53 immunostaining pattern. Infiltrating tumor cells show (A) p53 over-expression or (B) complete absence of p53 immunoreactivity. (C) The tumor cell nuclei show uniform and strong p53 expression, whereas the adjacent non-neoplastic epithelium (blue arrows) shows patchy positivity with weak-to-strong staining intensity. (D) The tumor cells are completely negative for $p 53$, whereas few lymphocytes (blue arrows) show weak nuclear immunoreactivity. (E) The tumor cells do not show any nuclear p16 expression except focal and weak cytoplasmic immunoreactivity. Staining method: A-E, polymer method. Original magnification: A, $40 x ; B, 100 x ; C, 200 x ; D, 200 x ; E, 150 x$.

inflammatory cells and stromal cells were patchily positive for p53 with variable staining intensity.

\section{Discussion}

Extension of endocervical adenocarcinoma to the uterine corpus is rarely encountered in daily practice. In addition to the shared histological features between endocervical and endometrial adenocarcinomas, extensive endomyometrial involvement of endocervical adenocarcinoma can lead to a misdiagnosis of primary endometrial adenocarcinoma with cervical stromal extension. An accurate and prudent differential diagnosis of uterine adenocarcinomas is crucial for treatment decision making, including the appropriate therapeutic option (e.g., radical vs. total hysterectomy), extent of lymph node sampling or dissection, and postoperative radiation therapy or chemotherapy (12).

GEA has a predilection to extend widely and deeply into the cervical stroma and myometrium and is usually associated with prominent stromal desmoplasia. However, in endometrial biopsy or curettage specimens, the amount of stromal tissue is often too small to evaluate whether the tumor is accompanied by desmoplastic reaction. Furthermore, because endometrial endometrioid carcinoma often shows an intracytoplasmic and intraglandular mucin, it is challenging to distinguish GEA from endometrial adenocarcinoma. Low-grade endometrioid carcinoma with mucinous differentiation typically displays mild nuclear pleomorphism, and it shows uniform and strong nuclear immunoreactivity for estrogen and progesterone receptors.

In addition to the hormone receptor positivity, aberrant nuclear $\beta$-catenin expression and loss of phosphatase and tensin homolog deletion on chromosome 10 (PTEN) expression favor a diagnosis of endometrial endometrioid carcinoma. High-grade endometrioid carcinoma with mucinous differentiation is frequently associated with microcystic, elongated, and fragmented (MELF) pattern of invasion; deep myometrial involvement; and LVSI. Prominent stromal desmoplasia and inflammatory infiltrates in association with MELF invasion pattern can raise the strong suspicion of GEA. However, high-grade endometrioid carcinoma of the endometrium consists of hypercellular, solid sheets of tumor cells with or without co-existing lowgrade endometrioid carcinoma components.

The presence of stromal histiocytic aggregations and intraepithelial neutrophils as well as endocervical-type intracytoplasmic mucin favor endometrial endometrioid carcinoma. Detection of mismatch repair (MMR) deficiency 
can also be useful to distinguish endometrioid carcinoma from GEA, as the latter does not exhibit an MMR-deficient phenotype but frequently harbors a TP53 mutation. According to the latest WHO Classification of Tumors of Female Reproductive Organs (17), the most frequent type of endocervical adenocarcinoma is UEA, followed by GEA.

Although cervicovaginal cytology screening and HPV vaccination have reduced the incidence rate of squamous cell carcinoma and HPVA, GEA has become relatively frequent. GEA shows significantly worse oncogenic behavior and clinical outcome in comparison to UEA, and it also has a different histogenesis and immunophenotype $(9,11,31,32)$. The IECC documented that apical mitotic figures and frequent apoptotic bodies that can be easily identified in UEA (2). In general, UEA consists of tumor cells with enlarged, elongated nuclei and mucin-poor, eosinophilic cytoplasm. It also contains less than $50 \%$ of cells with appreciable intracytoplasmic mucin $(2,33)$. In contrast, GEA consists of tumor cells with abundant clear or pale eosinophilic cytoplasm, distinct cytoplasmic borders, and low nuclear-cytoplasmic ratios. Further, it exhibits prominent stromal desmoplasia. As UEA is much more prevalent than GEA and an intracytoplasmic mucin within $50 \%$ can also be observed in UEA, it is sometimes challenging for pathologists to differentiate endocervical adenocarcinomas of UEA from those of GEA.

Wada et al. (32) analyzed diagnostically confusing cases of endocervical adenocarcinoma for reclassifying based on the latest WHO classification. They reported that the UEAlike pseudoendometrioid morphology of GEA can be induced by intracytoplasmic mucin depletion. Further, mucin-rich, GEA-like components can also exist in UEA (32). In the current study, patients 4-6 exhibited some mucin-poor glands and subtle desmoplastic stromal reaction, causing a confusion with UEA. Accordingly, an awareness of histological features and careful interpretation of immunostaining results should be used to ensure proper classification and avoid mistyping GEA as UEA. Clinicians should consider the possibility of GEA in cases presenting with voluminous, clear to pale eosinophilic cytoplasm; prominent stromal desmoplasia; non-block p16 positivity; and mutant p53 immunostaining pattern.

Considering the small size, the ovary is a relatively common site of metastasis (34). Metastatic ovarian neoplasms are thought to account for $10-30 \%$ of all ovarian malignancies, and the common primary sites include gastrointestinal tract, breast, pancreatobiliary organ, and other gynecological organs $(34,35)$. Most patients with metastatic ovarian neoplasms have known history of primary malignancy. However, some of them manifest only symptoms associated with an ovarian mass without any clinical history of malignancy. Although metastatic ovarian neoplasms usually present as bilateral ovarian masses that can provide a hint for distinguishing between metastasis and primary origin, imaging sometimes detects only unilateral ovarian mass or rather several masses in multiple organs, such as in patient 8 .

It is well-known that primary ovarian epithelial neoplasms, especially those showing mucinous differentiation, can be grossly and histologically mimicked by metastatic carcinoma, leading to misdiagnosis $(36,37)$. Grossly, primary ovarian mucinous tumors are typically large, unilateral, multilocular cystic lesions with a smooth capsule. Further, they histologically display an expansile/confluent glandular pattern or destructive/infiltrative stromal invasion (36). There are some histological features favoring a metastatic rather than a primary ovarian neoplasm. These include bilaterality, a nodular pattern of ovarian involvement, an infiltrative pattern of stromal invasion, microscopic surface deposits of tumor, and marked LVSI $(38,39)$. However, these features are not specific nor exclusive for metastasis.

The presence of benign and borderline components within the ovarian tumor can be a clue for the diagnosis of primary ovarian tumor. This is because primary ovarian carcinoma usually arises in association with a benign or borderline tumor. However, metastatic tumors can also sometimes include bland-appearing findings. Particularly, metastatic endocervical adenocarcinoma involving the ovary often has a deceptively bland-looking tumor area consisting of well-formed glands with mucinous epithelium, overlapping with those of primary ovarian benign or borderline mucinous tumor (35). This happens in other metastatic adenocarcinomas involving the ovary, especially those with mucinous differentiation, and is referred to as a maturation phenomenon $(35,38)$.

In addition to the above-mentioned difficulties, immunostaining is of no value to differentiate between cervical and ovarian origin, making it even harder for a pathologist to make a correct diagnosis. Both are expected to show cytokeratin (CK) 7 positivity and CK 20 negativity. In case of GEA, the most common HPV-independent adenocarcinoma, p16 immunostaining and HPV DNA detection are also not helpful. Despite these difficulties, it is crucial to correctly diagnose ovarian tumors as either primary or metastatic neoplasms because their clinical management and prognoses of may be significantly different from each other (36). Metastatic adenocarcinoma involving the ovary can morphologically mimic primary ovarian tumor; thus, pathologists should consider both the clinical and pathological context comprehensively.

As mentioned above, pancreatobiliary adenocarcinoma can also metastasize to the ovary. Notably, patients with pancreatobiliary neoplasm may not have apparent symptoms related to the primary site until late stage. As such, symptoms related to a metastatic ovarian mass or imaging findings of multicystic ovarian mass could be the first manifestation (38). 
We previously reported four cases of ovarian metastasis of pancreatobiliary origin (36). In one of the four cases, the primary tumor was diagnosed by follow-up investigation only 6 months after ovarian resection. Similar to metastatic endocervical adenocarcinoma involving the ovary, pancreatobiliary adenocarcinoma occasionally has a bland appearance and is deceptively similar to a primary ovarian mucinous tumor (35). In an ambiguous clinical setting, it is difficult to determine the primary origin as the ovary, pancreatobiliary tract, and cervix among ovarian mucinous tumors. In addition, the limited amount of specimen during the intraoperative frozen section examination makes it even harder to make a correct diagnosis of metastatic ovarian mass.

A previous study suggested an algorithm based on tumor size and laterality to determine if an ovarian tumor is primary or metastatic. Using the algorithm, they correctly classified all cases of ovarian metastasis originating from the pancreatobiliary tract (40). The study showed that bilaterality and smaller size favors a diagnosis of metastatic disease. Immunostaining is also of limited value and should be interpreted with caution only as an ancillary test. Both pancreatobiliary and gynecological neoplasms are commonly positive for CK 7 and stain variably for CK 20. However, recent studies reported that some tumors originating from the pancreatobiliary tract demonstrate loss of staining with deletions in pancreatic carcinoma 4 and over-expression of CK17 (41, 42). Extensive sampling for clues such as foci of destructive stromal invasion, LVSI, and bilaterality is the first step toward an accurate diagnosis. Further, a comprehensive clinicopathological evaluation with consideration of the possibility of metastasis is required.

Interestingly, we found a case of GEA with tubal intraepithelial metastases that were multifocal but were also microscopic. Although adnexal metastasis of cervical carcinoma is very uncommon, some studies have reported a secondary involvement of the fallopian tube in endocervical adenocarcinomas (29, 43). Reyes et al. (43) found eight cases of endocervical adenocarcinoma with metastatic tubal involvement. They reported that the tubal metastatic tumors often showed mucosal colonization mimicking a primary tubal lesion. Further, six of the eight tumors were unilateral and microscopically colonized pre-existing tubal epithelium simulating STIC.

We have also previously demonstrated that three endocervical adenocarcinomas (i.e., GEA, UEA, and mucinous-type adenocarcinoma) had tubal intraepithelial metastases only as an adnexal involvement (29). In this study, five of the eight patients with GEA had adnexal metastases, and one patient showed tubal intraepithelial metastasis alone. This patient showed no metastatic lesion in other anatomical locations including the ovary, bilateral pelvic lymph nodes, abdominal and pelvic peritoneum. This finding is in line with previous reports of tubal metastasis in early-stage cervical carcinoma $(44,45)$. Our observation of the endomyometrial involvement and intraluminal microscopic tumor emboli supports the idea of superficial luminal spread of tumor cells as one of the routes for tubal metastasis.

The most important differential diagnosis of tubal intraepithelial metastasis is STIC. STIC is histologically characterized by cellular crowding, stratification, loss of polarity, and enlarged nuclei with hyperchromasia, all of which can be observed in cases of tubal intraepithelial metastasis (29). In the presence of mucinous differentiation, the diagnosis of intraepithelial metastasis is favored. However, diagnosis based on morphological features can be difficult in the absence of these findings. In these cases, careful consideration of clinical history and the use of immunohistochemistry would be helpful. STIC can present as multifocal lesions but has a predilection for arising in the fimbrial portion. Thus, STIC is unlikely to involve both fimbrial and non-fimbrial portions. The absence of WT1 immunoreactivity, a serous cell lineage marker, rules out the possibility of STIC.

In summary, we described the detailed clinicopathological characteristics of eight cases of GEA misdiagnosed as endometrial, ovarian, or extragenital malignancies and mistyped as UEA. As already documented in the literature, GEA is an aggressive malignancy that presents frequently as an advanced-stage disease with various manifestations. When GEA involves the uterine corpus extensively and displays mucin-depleted glands in a pseudoendometrioid architecture, it closely resembles endometrial endometrioid carcinoma. A mucin-poor GEA having hyperchromatic, elongated nuclei, and apical mitotic figures and frequent apoptotic bodies makes it difficult to distinguish GEA from UEA based solely on histological features. A non-block p16 positivity and mutant p53 immunostaining pattern will be helpful in providing the diagnosis of GEA. Lastly, GEAs metastatic to the ovary mimic both primary ovarian mucinous carcinoma and metastatic adenocarcinoma of pancreatobiliary origin. Deceptively bland-looking areas within an ovarian tumor exacerbate the difficulty in identifying the primary origin. Given that accurate diagnosis in these cases is very important owing to its clinical implications in pathological staging, management, and prognosis, pathologists should comprehensively understand the clinical and histological findings of this entity.

\section{Conflicts of Interest}

None of the Authors have any conflicts of interest to declare regarding this study.

\section{Authors' Contributions}

All Authors substantial contributed to the conception and design of the study; acquisition, analysis, and interpretation of the data; drafting the manuscript; revising the manuscript critically for 
important intellectual content; and providing final approval of the version to be published.

\section{References}

1 Smith H, Tiffany M, Qualls C and Key C: The rising incidence of adenocarcinoma relative to squamous cell carcinoma of the uterine cervix in the United States - A 24-year population-based study. Gynecologic Oncology 78(2): 97-105, 2019. DOI: $10.1006 /$ gyno. 2000.5826

2 Stolnicu S, Barsan I, Hoang L, Patel P, Terinte C, Pesci A, AvielRonen S, Kiyokawa T, Alvarado-Cabrero I, Pike MC, Oliva E, Park KJ and Soslow RA: International Endocervical Adenocarcinoma Criteria and Classification (IECC): A new pathogenetic classification for invasive adenocarcinomas of the endocervix. Am J Surg Pathol 42(2): 214-226, 2018. PMID: 29135516. DOI: $10.1097 / \mathrm{PAS} .0000000000000986$

3 Talia KL and McCluggage WG: The developing spectrum of gastric-type cervical glandular lesions. Pathology 50(2): 122133, 2018. PMID: 29233547. DOI: 10.1016/j.pathol.2017.09.009

4 Kurman RJ, Carcangiu ML, Herrington CS and Young RH: WHO Classification of tumours of female reproductive organs. IARC, Lyon, 2014.

5 WHO Classification of Tumors Editorial Board: WHO Classification of tumours: Female genital tumours. IARC, Lyon, 2020.

6 Nishio S, Mikami Y, Tokunaga H, Yaegashi N, Satoh T, Saito M, Okamoto A, Kasamatsu T, Miyamoto T, Shiozawa T, Yoshioka Y, Mandai M, Kojima A, Takehara K, Kaneki E, Kobayashi H, Kaku T, Ushijima K and Kamura T: Analysis of gastric-type mucinous carcinoma of the uterine cervix - An aggressive tumor with a poor prognosis: A multi-institutional study. Gynecol Oncol 153(1): 13-19, 2019. PMID: 30709650. DOI: 10.1016/ j.ygyno.2019.01.022

7 Mikami Y: Gastric-type mucinous carcinoma of the cervix and its precursors - historical overview. Histopathology 76(1): 102111, 2020. PMID: 31846534. DOI: 10.1111/his.13993

8 Garg S, Nagaria TS, Clarke B, Freedman O, Khan Z, Schwock J, Bernardini MQ, Oza AM, Han K, Smith AC, Stockley TL and Rouzbahman M: Molecular characterization of gastric-type endocervical adenocarcinoma using next-generation sequencing. Mod Pathol 32(12): 1823-1833, 2019. PMID: 31308508. DOI: 10.1038/s41379-019-0305-x

9 Kojima A, Mikami Y, Sudo T, Yamaguchi S, Kusanagi Y, Ito M and Nishimura R: Gastric morphology and immunophenotype predict poor outcome in mucinous adenocarcinoma of the uterine cervix. Am J Surg Pathol 31(5): 664-672, 2007. PMID: 17460448. DOI: 10.1097/01.pas.0000213434.91868.b0

10 Park KJ, Kiyokawa T, Soslow RA, Lamb CA, Oliva E, Zivanovic O, Juretzka MM and Pirog EC: Unusual endocervical adenocarcinomas: an immunohistochemical analysis with molecular detection of human papillomavirus. Am J Surg Pathol 35(5): 633-646, 2011. PMID: 21490443. DOI: 10.1097/ PAS.0b013e31821534b9

11 Karamurzin YS, Kiyokawa T, Parkash V, Jotwani AR, Patel P, Pike MC, Soslow RA and Park KJ: Gastric-type endocervical adenocarcinoma: an aggressive tumor with unusual metastatic patterns and poor prognosis. Am J Surg Pathol 39(11): 1449-1457, 2015. PMID: 26457350. DOI: 10.1097/PAS.0000000000000532

12 Yemelyanova A, Vang R, Seidman JD, Gravitt PE and Ronnett BM: Endocervical adenocarcinomas with prominent endometrial or endomyometrial involvement simulating primary endometrial carcinomas: utility of HPV DNA detection and immunohistochemical expression of p16 and hormone receptors to confirm the cervical origin of the corpus tumor. Am J Surg Pathol 33(6): 914-924, 2009. PMID: 19295407. DOI: 10.1097/PAS.0b013e3181971fdd

13 Kamiya A, Ikura Y, Iizuka N, Yokokawa T, Kato H and Oishi T: Gastric-type endocervical adenocarcinoma with uterine corpus involvement mimicking primary endometrial carcinoma. J Obstet Gynaecol Res 45(7): 1414-1417, 2019. PMID: 31012190. DOI: $10.1111 /$ jog. 13973

14 Bae GE, Yoon N, Cho EY, Kim HS and Cho SY: Clinicopathological and molecular characteristics of mammary adenoid cystic carcinoma with adipocytic differentiation with emphasis on the identification of a novel BRAF mutation. Anticancer Res 39(1): 369-374, 2019. PMID: 30591482. DOI: 10.21873/anticanres.13121

15 Jung YY, Woo HY and Kim HS: Targeted genomic sequencing reveals novel TP53 in-frame deletion mutations leading to p53 overexpression in high-grade serous tubo-ovarian carcinoma. Anticancer Res 39(6): 2883-2889, 2019. PMID: 31177126. DOI: 10.21873/anticanres.13417

16 Kim HG, Park MS, Sung JY, Kim YW, Kim HS and Na K: Tumor-specific expression of insulin-like growth factor ii mRNA-binding protein 3 independently predicts worse survival of patients with adenocarcinoma of the ampulla of Vater. Anticancer Res 39(9): 4947-4955, 2019. PMID: 31519600. DOI: 10.21873/anticanres.13683

17 Jung H, Bae GE, Kim HM and Kim HS: Clinicopathological and molecular differences between gastric-type mucinous carcinoma and usual-type endocervical adenocarcinoma of the uterine cervix. Cancer Genomics Proteomics 17(5): 627-641, 2020. PMID: 32859641. DOI: 10.21873/cgp.20219

18 Kim JY, Kim SH and Kim HS: Promoter methylation downregulates osteoprotegerin expression in ovarian carcinoma. Anticancer Res 39(5): 2361-2367, 2019. PMID: 31092428. DOI: 10.21873/anticanres.13353

19 Koh HH, Lee MJ, Park NJ, Kim HS and Oh YL: Impact of implementing the Paris system for reporting urinary cytology: A single-institutional experience with emphasis on diagnostic yield of high-grade urothelial carcinoma and low-grade urothelial neoplasm. Anticancer Res 40(6): 3477-3484, 2020. PMID: 32487647. DOI: 10.21873/anticanres.14334

20 Park S, Cho EY, Oh YL, Park YH and Kim HS: Primary peritoneal high-grade serous carcinoma misinterpreted as metastatic breast carcinoma: a rare encounter in peritoneal fluid cytology. Anticancer Res 40(5): 2933-2939, 2020. PMID: 32366445. DOI: 10.21873/anticanres.14271

21 Woo HY, DO SI, Kim SH, Song SY and Kim HS: Promoter methylation down-regulates B-cell translocation gene 1 expression in breast carcinoma. Anticancer Res 39(10): 53615367, 2019. PMID: 31570430. DOI: 10.21873/anticanres.13729

$22 \mathrm{Kim} \mathrm{H}$, Choi S, Do SI, Lee SH, Yoon $\mathrm{N}$ and Kim HS: Clinicopathological characteristics of pleomorphic high-grade squamous intraepithelial lesion of the uterine cervix: a singleinstitutional series of 31 cases. Diagnostics (Basel) 10(8): 595, 2020. PMID: 32824242. DOI: 10.3390/diagnostics 10080595

23 Park S and Kim HS: Primary retroperitoneal mucinous carcinoma with carcinosarcomatous mural nodules: a case report with emphasis on its histological features and immunophenotype. 
Diagnostics (Basel) 10(8): 580, 2020. PMID: 32796551. DOI: 10.3390/diagnostics10080580

24 Kim HN, Woo HY, DO SI and Kim HS: Targeted sequencing of tubo-ovarian and peritoneal high-grade serous carcinoma with wild-type p53 immunostaining pattern. In Vivo 33(5): 14851492, 2019. PMID: 31471396. DOI: 10.21873/invivo.11628

25 Köbel M, Ronnett BM, Singh N, Soslow RA, Gilks CB and McCluggage WG: Interpretation of p53 immunohistochemistry in endometrial carcinomas: toward increased reproducibility. Int J Gynecol Pathol 38(Suppl 1): S123-S131, 2019. PMID: 29517499. DOI: 10.1097/PGP.0000000000000488

$26 \mathrm{Na} \mathrm{K}$, Sung JY and Kim HS: TP53 Mutation status of tuboovarian and peritoneal high-grade serous carcinoma with a wild-type p53 immunostaining pattern. Anticancer Res 37(12): 6697-6703, 2017. PMID: 29187446. DOI: 10.21873/anticanres. 12128

27 Chung T, DO SI, Na K, Kim G, Jeong YI, Kim YW and Kim HS: Stromal p16 overexpression in gastric-type mucinous carcinoma of the uterine cervix. Anticancer Res 38(6): 35513558, 2018. PMID: 29848709. DOI: 10.21873 /anticanres.12627

$28 \mathrm{Na}$ K, Sung JY and Kim HS: Clinicopathological characteristics of high-grade squamous intraepithelial lesions involving condyloma acuminatum. Anticancer Res 38(3): 1767-1774, 2018. PMID: 29491115. DOI: 10.21873/anticanres.12414

$29 \mathrm{Na} \mathrm{K}$ and Kim HS: Clinicopathological characteristics of fallopian tube metastases from primary endometrial, cervical, and nongynecological malignancies: a single institutional experience. Virchows Arch 471(3): 363-373, 2017. PMID: 28702779. DOI: $10.1007 / \mathrm{s} 00428-017-2186-\mathrm{z}$

$30 \mathrm{Na} \mathrm{K}$ and Kim HS: Clinicopathologic and molecular characteristics of mesonephric adenocarcinoma arising from the uterine body. Am J Surg Pathol 43(1): 12-25, 2019. PMID: 29189288. DOI: 10.1097/PAS.0000000000000991

31 Rodríguez-Carunchio L, Soveral I, Steenbergen RD, Torné A, Martinez S, Fusté P, Pahisa J, Marimon L, Ordi J and del Pino M: HPV-negative carcinoma of the uterine cervix: a distinct type of cervical cancer with poor prognosis. BJOG 122(1): 119-127, 2015. PMID: 25229645. DOI: 10.1111/1471-0528.13071

32 Wada T, Ohishi Y, Kaku T, Aman M, Imamura H, Yasutake N, Sonoda K, Kato K and Oda Y: Endocervical adenocarcinoma with morphologic features of both usual and gastric types: clinicopathologic and immunohistochemical analyses and highrisk HPV detection by in situ hybridization. Am J Surg Pathol 41(5): 696-705, 2017. PMID: 28296678. DOI: 10.1097/ PAS.0000000000000833

33 Young RH and Clement PB: Endocervical adenocarcinoma and its variants: their morphology and differential diagnosis. Histopathology 41(3): 185-207, 2002. PMID: 12207781. DOI: 10.1046/j.1365-2559.2002.01462.x

34 Shiono S, Saito T, Fujii H, Arakawa A, Nakamura T and Yao T: A case of Krukenberg carcinoma metastasized from colon cancer resembling mucinous cystadenocarcinoma of the ovary. Int J Clin Exp Pathol 7(1): 394-401, 2013. PMID: 24427362.

35 Alvarado-Cabrero I, Rodríguez-Gómez A, Castelan-Pedraza J and Valencia-Cedillo R: Metastatic ovarian tumors: a clinicopathologic study of 150 cases. Anal Quant Cytopathol Histpathol 35(5): 241-248, 2013. PMID: 24282903.

36 Park CK and Kim HS: Clinicopathological characteristics of ovarian metastasis from colorectal and pancreatobiliary carcinomas mimicking primary ovarian mucinous tumor.
Anticancer Res 38(9): 5465-5473, 2018. PMID: 30194204. DOI: 10.21873/anticanres.12879

37 Khunamornpong S, Siriaunkgul S, Suprasert P, Pojchamarnwiputh S, Na Chiangmai $\mathrm{W}$ and Young RH: Intrahepatic cholangiocarcinoma metastatic to the ovary: a report of 16 cases of an underemphasized form of secondary tumor in the ovary that may mimic primary neoplasia. Am J Surg Pathol 31(12): 1788-1799, 2007. PMID: 18043033. DOI: 10.1097/ PAS.0b013e3180674ded

38 McCluggage WG and Wilkinson N: Metastatic neoplasms involving the ovary: a review with an emphasis on morphological and immunohistochemical features. Histopathology 47(3): 231247, 2005. PMID: 16115224. DOI: 10.1111/j.1365-2559.2005. 02194.x

39 Meriden Z, Yemelyanova AV, Vang R and Ronnett BM: Ovarian metastases of pancreaticobiliary tract adenocarcinomas: analysis of 35 cases, with emphasis on the ability of metastases to simulate primary ovarian mucinous tumors. Am J Surg Pathol 35(2): 276-288, 2011. PMID: 21263249. DOI: 10.1097/PAS. 0b013e31820508d0

40 Yemelyanova AV, Vang R, Judson K, Wu LS and Ronnett BM: Distinction of primary and metastatic mucinous tumors involving the ovary: analysis of size and laterality data by primary site with reevaluation of an algorithm for tumor classification. Am J Surg Pathol 32(1): 128-138, 2008. PMID: 18162780. DOI: 10.1097/PAS.0b013e3180690d2d

$41 \mathrm{Ji}$ H, Isacson C, Seidman JD, Kurman RJ and Ronnett BM: Cytokeratins 7 and 20, Dpc4, and MUC5AC in the distinction of metastatic mucinous carcinomas in the ovary from primary ovarian mucinous tumors: Dpc4 assists in identifying metastatic pancreatic carcinomas. Int J Gynecol Pathol 21(4): 391-400, 2002. PMID: 12352188. DOI: 10.1097/00004347-20021000000009

42 Chu PG, Schwarz RE, Lau SK, Yen Y and Weiss LM: Immunohistochemical staining in the diagnosis of pancreatobiliary and ampulla of Vater adenocarcinoma: application of CDX2, CK17, MUC1, and MUC2. Am J Surg Pathol 29(3): 359-367, 2005. PMID: 15725805. DOI: 10.1097/ 01.pas.0000149708.12335.6a

43 Reyes C, Murali R and Park KJ: Secondary involvement of the adnexa and uterine corpus by carcinomas of the uterine cervix: a detailed morphologic description. Int J Gynecol Pathol 34(6): 551-563, 2015. PMID: 26166722. DOI: 10.1097/PGP. 0000000000000206

44 Deel CD, Allen RA, Holman LL and Zuna RE: Adenocarcinoma of the cervix involving the fallopian tube mucosa: report of a case. Diagn Pathol 11(1): 77, 2016. PMID: 27530463. DOI: 10.1186/s13000-016-0529-8

45 Gungor T, Altinkaya SO, Ozat M, Akbay S and Mollamahmutoglu L: Unusual form of superficial spreading squamous cell carcinoma of cervix involving the endometrium, bilateral tubes and ovaries: a case report with literature review. Arch Gynecol Obstet 283(2): 323-327, 2011. PMID: 20852876. DOI: $10.1007 / \mathrm{s} 00404-010-1672-1$

Received April 20, 2021

Revised May 8, 2021 Accepted May 10, 2021 